\title{
Performance Analysis of AF Relaying with Selection Combining in Nakagami- $m$ Fading
}

\author{
Dong Qin, Yuhao Wang, Senior Member, IEEE, Fuhui Zhou, Member, IEEE, and Kai-Kit Wong, Fellow, IEEE
}

\begin{abstract}
This paper investigates performance analysis of a selection combining scheme, which utilizes a variable gain amplify and forward (AF) relay over a Nakagami- $m$ fading channel. A selection combiner at a destination node chooses the better link between a relay channel and a direct channel. We derived exact closed-form expressions for moments of signal to noise ratio (SNR), ergodic capacity and average symbol error probability (SEP). Simulation examples confirm that our exact formulas offer a more accurate analysis tool for selection combining than other prevailing approximations without extra complexity. The derived expressions serve as a useful tool for system design due to their validity for any SNR and arbitrary system parameters.
\end{abstract}

Index Terms-selection combining, AF relaying, outage probability, Nakagami- $m$ fading, symbol error probability

\section{INTRODUCTION}

Internet of things covers many interdisciplinary issues, such as environmental monitoring, control of industrial processes, smart spaces and so on. As a complex technological innovation, internet of things confirms that future wireless communications are highly dense and heterogeneous. Dense networks make it possible for relay transmission, such as two-hop and multihop networks [1]-[3]. A relay node may forward a signal with either an amplify and forward (AF) or a decode and forward (DF) protocol. These two protocols have been well studied. For example, an approximate bit error probability was derived in [4] for a Rake receiver at relays and destination over multipath Nakagami- $m$ fading. A Turbo code was applied in [5] for asymptotic outage probability in relay selection networks. The distributed relay processing, ideal centralized maximal ratio combining and centralized maximal ratio combining relay processing were employed in [6], which introduced Nakagami and Gamma distribution for approximate bit error rate of binary phase shift keying. Incremental DF and AF relaying were considered in [7] for analyzing the diversity multiplexing tradeoff. Orthogonal space time block coding was assumed in [8] for deriving closed formula of higher moments of end-to-end signal to noise ratio (SNR).

Diversity technique aims at a performance improvement of a wireless system. Among all different diversity schemes, selection combining may be the simplest one. The importance of selection combining has motivated numerous researches.

This work was supported by the National Natural Science Foundation of China (Grant Nos. 61761030, 61661028, 61701214, 61861017), by the China Postdoctoral Science Foundation (Grant No. 2017M622103).

Dong Qin, Yuhao Wang and Fuhui Zhou are with the Information Engineering School, Nanchang University, Nanchang, China (e-mail: qindong@seu.edu.cn).

K.-K. Wong is with the Department of Electronic and Electrical Engineering, University College London, United Kingdom.
Selection combining of direct and relayed paths in space shift keying was analyzed in [9]. A bit error rate based selection combining was proposed in [10], which calculated bit error rate of each branch and selected the branch with the minimum bit error rate. A single threshold was employed to select retransmitting relays in [11]. Exact bit error rate formulas of digital modulation was derived in [12] when an arbitrary number of relays used selection combining. In [13], selection combining was employed in cyclic prefixed single carrier DF relaying protocol and the transmit antenna selection and receive selection combining was investigated in [14]. An arbitrary fading parameter $m$ was considered in [15], [16] for DF relaying systems while Rayleigh fading was studied in [17]-[19] for AF relaying.

Performance analysis of selection combining in a Nakagami channel was recently reported but mostly for the case of integer $m$ [13], [14], [20]. When non integer $m$ is encountered, infinite series expansion has to be exploited for numerical evaluation, whose exact analytical evaluation appears to be difficult [16]. Some researchers only provided a loose lower or upper bound by simplification [21]. Infinite series always requires truncated partial summation, whose numbers of terms rely on a precision threshold. For comparison, the previous works are summarized in Tab.I.

To improve performance evaluation accuracy and reduce the calculation complexity, this paper investigates performance analysis of an AF relay system with selection combining over Nakagami- $m$ fading channel, where $m$ is a positive number or a non negative number plus one half. Although maximal ratio combining is the optimal combining technique, it requires computing the optimal weight for each branch. Selection combining may be the simplest combining scheme, which selects the signal with the largest SNR out of multiple branches and ignores the remaining branches. The decision variable is computed between the direct link and the relaying link and the one with a larger magnitude is chosen for selection combining. In practice, the selection combining technology has been used in many scenarios, such as the internet of things domain [3] and power line communications [22]. The exact higher order moments of the end to end SNR, ergodic capacity and average symbol error probability (SEP) formulas are obtained. Different from the additive white Gaussian noise (AWGN) assumption in most studies, our problem formulation is also extended to an additive white generalized Gaussian noise (AWGGN) environment, which is a more general type of noise model. The exact SEP formula subject to AWGGN implies that one can obtain the error performance in a more general scenario, such as Laplace noise and uniformly distributed 
Table I

A SUMMARY OF PREVIOUS WORKS

\begin{tabular}{|c|c|c|c|c|}
\hline References & Relaying protocol & Parameter $m$ & Performance analysis & Performance expression \\
\hline [9], [10] & DF & $m=1$ (Rayleigh fading) & Bit error rate & Approximation \\
\hline$[11],[12]$ & DF & $m=1$ (Rayleigh fading) & Bit error rate & Exact value \\
\hline [13] & DF & Integer & Outage probability & Asymptotic value \\
\hline$[14]$ & $\mathrm{DF}$ & Integer & Outage probability, ergodic capacity and SEP & Exact value \\
\hline$[15]$ & DF & Arbitrary & Outage probability & Exact value \\
\hline [16] & $\mathrm{DF}$ & Arbitrary & Outage probability, ergodic capacity and SEP & Infinite series expansion \\
\hline$[17]-[19]$ & $\mathrm{AF}$ & $m=1$ (Rayleigh fading) & Bit error rate & Exact value \\
\hline$[20]$ & $\mathrm{AF}$ & Integer & Conditional error rate of binary signaling & Exact value \\
\hline$[21]$ & $\mathrm{AF}$ & Arbitrary & SEP & Upper bound and asymptotic value \\
\hline
\end{tabular}

Table II

A SUMMARY OF ABBREVIATIONS

\begin{tabular}{|c|c|}
\hline Abbreviation & Full form \\
\hline AF & Amplify and forward \\
\hline AWGGN & Additive white generalized Gaussian noise \\
\hline AWGN & Additive white Gaussian noise \\
\hline DF & Decode and forward \\
\hline PAM & Pulse amplitude modulation \\
\hline QAM & Quadrature amplitude modulation \\
\hline SEP & Symbol error probability \\
\hline SNR & Signal to noise ratio \\
\hline
\end{tabular}

Table III

MATHEMATICAL SYMBOLS

\begin{tabular}{|c|c|}
\hline Notation & Description \\
\hline$\lfloor\cdot\rfloor$ & Floor function \\
\hline$C \cdot$ & Binomial coefficient \\
\hline$e r f c(\cdot)$ & Complementary error function \\
\hline $\mathrm{E}(\cdot)$ & Expectation operator \\
\hline$p F_{q}(\cdot)$ & The upper incomplete gamma function \\
\hline$\Gamma(\cdot)$ & Meijer's $G$ function \\
\hline$\Gamma(\cdot, \cdot)$ & Fox's $H$ function \\
\hline$G(\cdot)$ & Bivariate Fox's function \\
\hline$H(\cdot)$ & Confluent hypergeometric function \\
\hline$H(x, y)$ & Gauss $Q$ function \\
\hline$K_{\nu}(\cdot)$ & The $\nu$ th order modified Bessel function of the second kind \\
\hline$\Psi(\cdot, \cdot ; \cdot)$ & Generalized $Q$ function \\
\hline$Q(\cdot)$ & \\
\hline$Q_{\alpha}(\cdot)$ & \\
\hline
\end{tabular}

noise. These formulas provide an insight into practical relay system design. Finally, the abbreviations and mathematical symbols are summarized in Tabs.II and III.

\section{SySTEM ModeL}

Consider a relay system consisting of a source node $S$, an AF relay node $\mathrm{R}$, and a destination node D, as shown in Fig.1. $\mathrm{S}$ transmits information to $\mathrm{D}$ directly or via $\mathrm{R}$ during two phases. In the first phase, $\mathrm{S}$ sends its signals while $\mathrm{R}$ and $\mathrm{D}$ listen. In the second phase, $\mathrm{S}$ stays silent while $\mathrm{R}$ forwards the amplified signals to $\mathrm{D}$ if the relay link is beneficial. We assume that all channels experience independent Nakagami$m$ fading, where $m$ is a positive number or a non negative number plus one half. Let $p_{s}$ and $p_{r}$ be the transmit powers of $\mathrm{S}$ and R, respectively. Denote by $h_{s}, h_{r}$ and $h_{d}$ the channel coefficients of the $\mathrm{S} \rightarrow \mathrm{R}, \mathrm{R} \rightarrow \mathrm{D}$ and $\mathrm{S} \rightarrow \mathrm{D}$ links, respectively. In addition, $\sigma_{r}^{2}$ and $\sigma_{d}^{2}$ represents noise variances at $\mathrm{R}$ and $\mathrm{D}$, respectively. Thus, the instantaneous SNR of the direct link

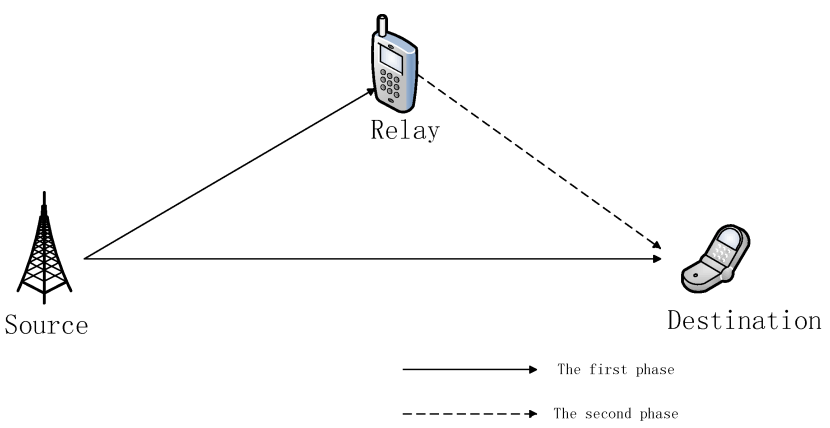

Figure 1. System model

and the SNR of the relay link are, respectively, given by [20]

$$
\begin{aligned}
\gamma_{1} & =\frac{p_{s}\left|h_{d}\right|^{2}}{\sigma_{d}^{2}} \\
\gamma_{2} & =\frac{\frac{p_{s}\left|h_{s}\right|^{2} p_{r}\left|h_{r}\right|^{2}}{\sigma_{r}^{2} \sigma_{d}^{2}}}{\frac{p_{s}\left|h_{s}\right|^{2}}{\sigma_{r}^{2}}+\frac{p_{r}\left|h_{r}\right|^{2}}{\sigma_{d}^{2}}}
\end{aligned}
$$

Assuming that $\mathrm{D}$ combines the received signals from the $\mathrm{S} \rightarrow \mathrm{D}$ and $\mathrm{S} \rightarrow \mathrm{R} \rightarrow \mathrm{D}$ links by selection combining, the end to end SNR at D is given by [20]

$$
\gamma=\max \left(\gamma_{1}, \gamma_{2}\right)
$$

The corresponding cumulative distribution function (CDF) $F_{\gamma}(z)$ is given by [20]

$$
F_{\gamma}(z)=F_{\gamma_{1}}(z) F_{\gamma_{2}}(z)
$$

\section{Performance AnAlysis}

\section{A. Outage Probability}

1) $m$ is a positive number: Since $h_{s}, h_{r}$ and $h_{d}$ are modeled as Nakagami- $m$ random variables, the CDFs of $\gamma_{s}$ and $\gamma_{r}$ are, respectively, given by [20]

$$
\begin{aligned}
F_{\gamma_{1}}(x)=1 & -\frac{\Gamma\left(m_{d}, \beta_{d} x\right)}{\Gamma\left(m_{d}\right)} \\
F_{\gamma_{2}}(y)=1 & -\frac{2 e^{-\left(\beta_{s}+\beta_{r}\right) y}}{\Gamma\left(m_{r}\right)} \sum_{l_{1}=0}^{m_{s}-1} \sum_{k=0}^{l_{1}} \sum_{l_{2}=0}^{m_{r}-1} \frac{C_{l_{1}}^{k} C_{m_{r}-1}^{l_{2}}}{l_{1} !} \\
& \times \beta_{s}^{l_{1}+\frac{1-k+l_{2}}{2}} \beta_{r}^{m_{r}-\frac{1-k+l_{2}}{2}} y^{l_{1}+m_{r}} \\
& \times K_{k-1-l_{2}}\left(2 \sqrt{\beta_{s} \beta_{r}} y\right)
\end{aligned}
$$


where $m_{s}, m_{r}$ and $m_{d}$ are the shape parameters of channel coefficients $h_{s}, h_{r}$ and $h_{d}$, respectively. Moreover, $\beta_{s}=$ $m_{s} \sigma_{r}^{2} /\left[p_{s} \mathrm{E}\left(\left|h_{s}\right|^{2}\right)\right], \beta_{r}=m_{r} \sigma_{d}^{2} /\left[p_{r} \mathrm{E}\left(\left|h_{r}\right|^{2}\right)\right]$ and $\beta_{d}=$ $m_{d} \sigma_{d}^{2} /\left[p_{s} \mathrm{E}\left(\left|h_{d}\right|^{2}\right)\right]$ denote the corresponding scale parameters, where $\mathrm{E}(\cdot)$ is the expectation operator, $C$. stands for the binomial coefficient and $K_{\nu}(\cdot)$ is the $\nu$ th order modified Bessel function of the second kind [23, eq.(8.432.1)]. Furthermore, $\Gamma(\cdot)$ is the gamma function $[23$, eq. $(8.310 .1)]$ and $\Gamma(\cdot, \cdot)$ is the upper incomplete gamma function [23, eq.(8.350.2)].

2) $m$ is a non negative number plus one half: The CDF of $\gamma_{1}$ is the same as eq.(5) but the CDF of $\gamma_{2}$ is written as follows [24]

$$
\begin{aligned}
F_{\gamma_{2}}(y)= & -\frac{\sqrt{\pi} e^{-\left(\sqrt{\beta_{s}}+\sqrt{\beta_{r}}\right)^{2} y}\left(\sqrt{\beta_{s}}+\sqrt{\beta_{r}}\right)}{\Gamma\left(m_{s}\right) \Gamma\left(m_{r}\right)} \sum_{k_{1}=0}^{\left\lfloor m_{s}\right\rfloor} \sum_{k_{2}=0}^{\left\lfloor m_{r}\right\rfloor} \\
& \frac{\left(\left\lfloor m_{s}\right\rfloor+k_{1}\right) !\left(\left\lfloor m_{r}\right\rfloor+k_{2}\right) ! \beta_{s}^{\frac{m_{s}}{2}-\frac{1}{4}-\frac{k_{1}}{2}} \beta_{r}^{\frac{m_{r}}{2}-\frac{1}{4}-\frac{k_{2}}{2}}}{4^{k_{1}+k_{2}} k_{1} ! k_{2} !\left(\left\lfloor m_{s}\right\rfloor-k_{1}\right) !\left(\left\lfloor m_{r}\right\rfloor-k_{2}\right) !} \\
& \times y^{\frac{m_{s}-k_{1}+m_{r}-k_{2}}{2}} \Psi\left[\frac{3-\left(m_{s}-k_{1}+m_{r}-k_{2}\right)}{2}, \frac{3}{2} ;\right. \\
& \left.\left(\sqrt{\beta_{s}}+\sqrt{\beta_{r}}\right)^{2} y\right]
\end{aligned}
$$

where $\lfloor\cdot\rfloor$ is the floor function and $\Psi(\cdot, \cdot ; \cdot)$ is the confluent hypergeometric function defined in [23, eq.(9.210.2)].

Then, the outage probability of the end to end SNR $\gamma$ is given by

$$
P_{\text {out }}=\operatorname{Pr}\left(\gamma \leq \gamma_{t h}\right)=F_{\gamma}\left(\gamma_{t h}\right)
$$

where $\gamma_{t h}$ is a preset threshold. Substituting the corresponding $F_{\gamma_{1}}(x)$ and $F_{\gamma_{2}}(y)$ into eq.(4) yields the exact outage probability $P_{\text {out }}$.

\section{B. Moments of SNR}

In selection combining, the destination chooses a larger magnitude as the decision variable between the direct link and the indirect link. Again, we consider the following two cases

1) $m$ is a positive number: In order to calculate the higher order moment, the CDF of the equivalent SNR is rewritten as

$$
\begin{aligned}
F_{\gamma}(z) & =F_{\gamma_{1}}(z) F_{\gamma_{2}}(z) \\
& =1-\bar{F}_{\gamma_{1}}(z)-\bar{F}_{\gamma_{2}}(z)+\bar{F}_{\gamma_{1}}(z) \bar{F}_{\gamma_{2}}(z)
\end{aligned}
$$

where $\bar{F}(\cdot)=1-F(\cdot)$ is the complementary CDF. Taking the expectation of $\gamma^{n}$ over the distribution of $F_{\gamma}(z)$ via integration by parts, the higher order moment is obtained as

$$
\begin{aligned}
\mathrm{E}\left(\gamma^{n}\right)= & \int_{0}^{\infty} n\left[1-F_{\gamma}(z)\right] z^{n-1} d z \\
= & \int_{0}^{\infty} n\left[\bar{F}_{\gamma_{1}}(z)+\bar{F}_{\gamma_{2}}(z)-\bar{F}_{\gamma_{1}}(z) \bar{F}_{\gamma_{2}}(z)\right] \\
& \times z^{n-1} d z
\end{aligned}
$$

The integral of the first two terms in (9) can be obtained from the integral table identity [23, eq.(6.455)]. Since the fading parameter is a positive number, one can expand $\bar{F}_{\gamma_{1}}(z)$ using the series identity [23, eq.(8.352.2)]. By substituting the three terms in (9), the moments of the SNR $\gamma$ are given by (10), where ${ }_{p} F_{q}\left(\alpha_{1}, \ldots, \alpha_{p} ; \beta_{1}, \ldots, \beta_{q} ; z\right)$ is called a generalized hypergeometric series [23, eq.(9.14)].

2) $m$ is a non negative number plus one half: Likewise, the higher-order moments are computed by integration by parts. The second and third terms of (9) involve the integral of the confluent hypergeometric function, which can be solved using [23, eq.(7.621.6)]. The moments of the SNR $\gamma$ are given by (11).

\section{Ergodic Capacity}

1) $m$ is a positive number: If the magnitude of the received signal in the relay link is greater, then the destination instructs the relay to participate in cooperation, otherwise it does not. Using the rule of integration by parts, the ergodic capacity is written as

$$
\begin{aligned}
\mathrm{E}\left[\frac{1}{2} \log _{2}(1+\gamma)\right]= & \int_{0}^{\infty} \frac{1-F_{\gamma}(z)}{2 \ln 2(1+z)} d z \\
= & \int_{0}^{\infty} \frac{\bar{F}_{\gamma_{1}}(z)+\bar{F}_{\gamma_{2}}(z)}{2 \ln 2(1+z)} d z \\
& -\int_{0}^{\infty} \frac{\bar{F}_{\gamma_{1}}(z) \bar{F}_{\gamma_{2}}(z)}{2 \ln 2(1+z)} d z
\end{aligned}
$$

When the first term of (12) is tackled, one kind of integral appears as

$$
J_{1}=\int_{0}^{\infty} \frac{\Gamma\left(m_{d}, \beta_{d} x\right)}{1+x} d x
$$

Here, some constant coefficients are omitted. Next, we rewrite the Gamma and fractional functions in terms of Meijer's $G$ function as follows

$$
\begin{aligned}
& \Gamma\left(m_{d}, \beta_{d} x\right)=G_{1,2}^{2,0}\left[\begin{array}{l|c}
\beta_{d} x & 1 \\
m_{d}, 0
\end{array}\right] \\
& \frac{1}{1+x}=G_{1,1}^{1,1}\left[x \mid \begin{array}{l}
0 \\
0
\end{array}\right]
\end{aligned}
$$

where $G(\cdot)$ is the Meijer's $G$ function [23, eq.(9.301)]. Using [23, eq.(7.811.1)], $J_{1}$ is expressed as a Meijer's $G$ function. When the second term of (12) is tackled, one kind of integral appears as

$$
J_{2}=\int_{0}^{\infty} \frac{e^{-\left(\beta_{s}+\beta_{r}\right) x}}{1+x} x^{l_{1}+m_{r}} K_{k-1-l_{2}}\left(2 \sqrt{\beta_{s} \beta_{r}} x\right) d x
$$

Likewise, the exponential function is rewritten as

$$
e^{-\left(\beta_{s}+\beta_{r}\right) x}=H_{0,1}^{1,0}\left[\left(\beta_{s}+\beta_{r}\right) x \mid \begin{array}{c}
- \\
(0,1)
\end{array}\right]
$$

where $H(\cdot)$ is the Fox's $H$ function [25, eqs.(2.9.4),(1.1.1)]. Using [26, eq.(2.6.4)] yields the result $J_{2}$ in (16). Adding the constant coefficients and combining $J_{1}$ and $J_{2}$, the ergodic capacity is given by $(18)$, where $H(x, y)$ is the bivariate Fox's function [26, eq.(2.2.1)].

2) $m$ is a non negative number plus one half: Similarly, based on the bivariate Meijer's function, when $m$ is a non negative number plus one half, the ergodic capacity is given by (19). 


$$
\begin{aligned}
\mathrm{E}\left(\gamma^{n}\right)= & \frac{\Gamma\left(m_{d}+n\right)}{\Gamma\left(m_{d}\right) \beta_{d}^{n}}+\frac{8 n \sqrt{\pi} \beta_{r}^{m_{r}}}{\Gamma\left(m_{r}\right)} \sum_{l_{1}=0}^{m_{s}-1} \sum_{k=0}^{l_{1}} \sum_{l_{2}=0}^{m_{r}-1} \frac{C_{l_{1}}^{k} C_{m_{r}-1}^{l_{2}} 4^{l_{2}-k} \beta_{s}^{l_{1}+1-k+l_{2}} \Gamma\left(1+l_{2}-k+l_{1}+n+m_{r}\right)}{l_{1} ! \Gamma\left(\frac{1}{2}+l_{1}+n+m_{r}\right)} \\
& \times \Gamma\left(k-1-l_{2}+l_{1}+n+m_{r}\right)\left(\sqrt{\beta_{s}}+\sqrt{\beta_{r}}\right)^{2\left(k-1-l_{2}-l_{1}-n-m_{r}\right)} \\
& \times{ }_{2} F_{1}\left[\frac{3}{2}-k+l_{2}, 1+l_{2}-k+l_{1}+n+m_{r} ; \frac{1}{2}+l_{1}+n+m_{r} ;\left(\frac{\sqrt{\beta_{s}}-\sqrt{\beta_{r}}}{\sqrt{\beta_{s}}+\sqrt{\beta_{r}}}\right)^{2}\right] \\
& -\frac{\sqrt{\pi} n}{2 \Gamma\left(m_{r}\right)} \sum_{l_{1}=0}^{m_{s}-1} \sum_{k=0}^{l_{1}} \sum_{l_{2}=0}^{m_{r}-1} \sum_{l_{3}=0}^{m_{d}-1} \frac{C_{l_{1}}^{k} C_{m_{r}-1}^{l_{2}} 4^{k-l_{2}} \beta_{s}^{l_{1}} \beta_{r}^{m_{r}+k-1-l_{2}} \beta_{d}^{l_{3}} \Gamma\left(l_{1}+m_{r}+n+l_{3}+k-1-l_{2}\right)}{l_{1} !\left(\beta_{s}+\beta_{r}+\beta_{d}+2 \sqrt{\beta_{s} \beta_{r}}\right)^{l_{1}+m_{r}+n+l_{3}+k-1-l_{2}} \Gamma\left(l_{1}+m_{r}+n+l_{3}+\frac{1}{2}\right)} \\
& \times \Gamma\left(l_{1}+m_{r}+n+l_{3}-k+1+l_{2}\right) \\
& \times{ }_{2} F_{1}\left[l_{1}+m_{r}+n+l_{3}+k-1-l_{2}, k-l_{2}-\frac{1}{2} ; l_{1}+n+m_{r}+l_{3}+\frac{1}{2} ; \frac{\left(\sqrt{\beta_{s}}-\sqrt{\beta_{r}}\right)^{2}+\beta_{d}}{\left(\sqrt{\beta_{s}}+\sqrt{\beta_{r}}\right)^{2}+\beta_{d}}\right]
\end{aligned}
$$

$$
\begin{aligned}
& \mathrm{E}\left(\gamma^{n}\right)=\frac{\Gamma\left(m_{d}+n\right)}{\Gamma\left(m_{d}\right) \beta_{d}^{n}}+\frac{\sqrt{\pi}}{\Gamma\left(m_{s}\right) \Gamma\left(m_{r}\right)(n-1) !} \sum_{k_{1}=0}^{\left\lfloor m_{s}\right\rfloor} \sum_{k_{2}=0}^{\left\lfloor m_{r}\right\rfloor} \frac{\left(\left\lfloor m_{s}\right\rfloor+k_{1}\right) !\left(\left\lfloor m_{r}\right\rfloor+k_{2}\right) ! \beta_{s}^{\frac{m_{s}}{2}-\frac{1}{4}-\frac{k_{1}}{2}} \beta_{r}^{\frac{m_{r}}{2}-\frac{1}{4}-\frac{k_{2}}{2}}}{4^{k_{1}+k_{2}} k_{1} ! k_{2} !\left(\left\lfloor m_{s}\right\rfloor-k_{1}\right) !\left(\left\lfloor m_{r}\right\rfloor-k_{2}\right) !} \\
& \times\left(\sqrt{\beta_{s}}+\sqrt{\beta_{r}}\right)^{1-m_{s}+k_{1}-m_{r}+k_{2}-2 n} \Gamma\left(\frac{m_{s}-k_{1}+m_{r}-k_{2}}{2}+n\right) \Gamma\left(\frac{m_{s}-k_{1}+m_{r}-k_{2}-1}{2}+n\right) \\
& -\frac{4 \pi\left(\sqrt{\beta_{s}}+\sqrt{\beta_{r}}\right)}{\Gamma\left(m_{s}\right) \Gamma\left(m_{r}\right)(n-1) !} \sum_{k_{1}=0}^{\left\lfloor m_{s}\right\rfloor} \sum_{k_{2}=0}^{\left\lfloor m_{r}\right\rfloor} \frac{\left(\left\lfloor m_{s}\right\rfloor+k_{1}\right) !\left(\left\lfloor m_{r}\right\rfloor+k_{2}\right) ! \beta_{s}^{\frac{m_{s}}{2}-\frac{1}{4}-\frac{k_{1}}{2}} \beta_{r}^{\frac{m_{r}}{2}-\frac{1}{4}-\frac{k_{2}}{2}}}{4^{k_{1}+k_{2}} k_{1} ! k_{2} !\left(\left\lfloor m_{s}\right\rfloor-k_{1}\right) !\left(\left\lfloor m_{r}\right\rfloor-k_{2}\right) !} \\
& \times\left[2\left(\sqrt{\beta_{s}}+\sqrt{\beta_{r}}\right)\right]^{k_{1}+k_{2}-2 n-m_{s}-m_{r}-2 m_{d}}\left\{\left[2\left(\sqrt{\beta_{s}}+\sqrt{\beta_{r}}\right)\right]^{2 m_{d}} \Gamma\left(2 n+m_{s}+m_{r}-k_{1}-k_{2}-1\right)\right. \\
& -\frac{n ! \beta_{d}^{m_{d}} \Gamma\left(2 n+m_{s}+m_{r}+2 m_{d}-k_{1}-k_{2}-1\right)}{\Gamma\left(1+m_{d}\right) \Gamma\left(1+m_{d}+n\right)}{ }_{3} F_{2}\left[m_{d}, \frac{m_{s}+m_{r}-k_{1}-k_{2}-1}{2}+n+m_{d},\right. \\
& \left.\left.\frac{m_{s}+m_{r}-k_{1}-k_{2}}{2}+n+m_{d} ; 1+m_{d}, 1+m_{d}+n ; \frac{-\beta_{d}}{\left(\sqrt{\beta_{s}}+\sqrt{\beta_{r}}\right)^{2}}\right]\right\}
\end{aligned}
$$

$$
\begin{aligned}
& \mathrm{E}\left[\frac{1}{2} \log _{2}(1+\gamma)\right]=\frac{1}{2 \ln 2 \Gamma\left(m_{d}\right)} G_{2,3}^{3,1}\left(\beta_{d} \mid \begin{array}{c}
0,1 \\
m_{d}, 0,0
\end{array}\right)+\frac{1}{\ln 2 \Gamma\left(m_{r}\right)} \sum_{l_{1}=0}^{m_{s}-1} \sum_{k=0}^{l_{1}} \sum_{l_{2}=0}^{m_{r}-1} \frac{C_{l_{1}}^{k} C_{m_{r}-1}^{l_{2}}}{l_{1} !} \beta_{s}^{\frac{l_{1}+l_{2}-m_{r}-k}{2}} \beta_{r}^{\frac{m_{r}+k-l_{1}-l_{2}}{2}-1} \\
& \times H_{2,(0: 1), 0,(1: 1)}^{2,0,1,1,1}\left[\begin{array}{c|c}
\frac{\left(\beta_{s}+\beta_{r}\right)^{2}}{\beta_{s} \beta_{r}} & \left(\frac{l_{1}+m_{r}+k-l_{2}}{2}, 1\right),\left(\frac{l_{1}+m_{r}-k+l_{2}}{2}+1,1\right) \\
\frac{\beta_{s} \beta_{r}}{2} & -;(0,2) \\
& (0,2) ;(0,2)
\end{array}\right] \\
& -\frac{1}{\ln 2 \Gamma\left(m_{r}\right)} \sum_{l_{1}=0}^{m_{s}-1} \sum_{k=0}^{l_{1}} \sum_{l_{2}=0}^{m_{r}-1} \sum_{l_{3}=0}^{m_{d}-1} \frac{C_{l_{1}}^{k} C_{m_{r}-1}^{l_{2}}}{l_{1} ! l_{3} !} \beta_{d}^{l_{3}} \beta_{s}^{\frac{l_{1}-k+l_{2}-m_{r}-l_{3}}{2}} \beta_{r}^{\frac{m_{r}+k-l_{2}-l_{1}-l_{3}}{2}-1} \\
& \times H_{2,(0: 1), 0,(1: 1)}^{2,0,1,1}\left[\begin{array}{c|c}
\frac{\left(\beta_{s}+\beta_{r}+\beta_{d}\right)^{2}}{\beta_{s} \beta_{r}} & \left(\frac{l_{1}+m_{r}+l_{3}+k-l_{2}}{2}, 1\right),\left(\frac{l_{1}+m_{r}+l_{3}-k+l_{2}}{2}+1,1\right) \\
\frac{\beta_{s} \beta_{r}}{} & -;(0,2) \\
- & (0,2) ;(0,2)
\end{array}\right]
\end{aligned}
$$




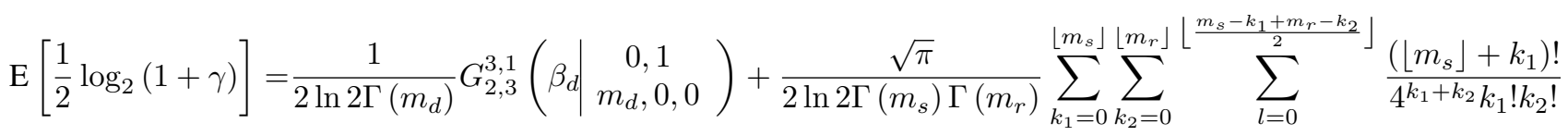

$$
\begin{aligned}
& \times \frac{\left(\left\lfloor m_{r}\right\rfloor+k_{2}\right) ! \beta_{s}^{\frac{m_{s}}{2}-\frac{1}{4}-\frac{k_{1}}{2}} \beta_{r}^{\frac{m_{r}}{2}-\frac{1}{4}-\frac{k_{2}}{2}}\left(m_{s}-k_{1}+m_{r}-k_{2}\right) !(-1)^{l}}{\left(\left\lfloor m_{s}\right\rfloor-k_{1}\right) !\left(\left\lfloor m_{r}\right\rfloor-k_{2}\right) ! 2^{2 l} l !\left(m_{s}-k_{1}+m_{r}-k_{2}-2 l\right) !} \\
& \times\left(\sqrt{\beta_{s}}+\sqrt{\beta_{r}}\right)^{k_{1}-m_{s}+k_{2}-m_{r}+1}\left\{G_{2,3}^{3,1}\left[\left(\sqrt{\beta_{s}}+\sqrt{\beta_{r}}\right)^{2} \mid \begin{array}{c}
0,1 \\
m_{s}-k_{1}+m_{r}-k_{2}-l-\frac{1}{2}, 0,0
\end{array}\right]\right. \\
& \left.-\frac{1}{\Gamma\left(m_{d}\right) \beta_{d}} G_{2,(1: 1), 1,(2: 1)}^{2,0,1,2,1}\left[\begin{array}{c|c}
\frac{\left(\sqrt{\beta_{s}}+\sqrt{\beta_{r}}\right)^{2}}{\beta_{d}} & m_{d}+1,1 \\
\frac{1}{\beta_{d}} & 1 ; 0 \\
2 & m_{s}-k_{1}+m_{r}-k_{2}-l-\frac{1}{2}, 0 ; 0
\end{array}\right]\right\}
\end{aligned}
$$

\section{Average SEP}

1) $m$ is a positive number: The average SEP conditioned on the instantaneous SNR is given by

$$
P_{e}=\mathrm{E}\left[a Q(\sqrt{b \gamma})-c Q^{2}(\sqrt{b \gamma})\right]
$$

where $a, b$ and $c$ are constants dependent on the modulation type and $Q(\cdot)$ is the Gauss $Q$ function. Applying the rule of integration by parts, $P_{e}$ can be rewritten as

$$
\begin{aligned}
P_{e}= & \int_{0}^{\infty} \frac{a \sqrt{b} e^{-\frac{b x}{2}}}{2 \sqrt{2 \pi x}} F_{\gamma}(x) d x \\
& -\int_{0}^{\infty} \frac{c \sqrt{2 b} e^{-\frac{b x}{2}}}{4 \sqrt{\pi x}} \operatorname{erfc}\left(\sqrt{\frac{b x}{2}}\right) F_{\gamma}(x) d x
\end{aligned}
$$

where $\operatorname{erfc}(\cdot)$ is the complementary error function. The first and second terms of (21) are denoted by $P_{e 1}$ and $P_{e 2}$, respectively. Let us check $P_{e 1}$ first. Using [23, eq.(6.455.1)], the average SEP $P_{e 1}$ is given by (22).

Analyzing $P_{e 2}$ is challenging due to the complex nature of the complementary error function. One type of integral $J_{3}$ appears as

$$
\begin{aligned}
J_{3}= & \int_{0}^{\infty} \operatorname{erfc}\left(\sqrt{\frac{b x}{2}}\right) e^{-\left(\frac{b}{2}+\beta_{s}+\beta_{r}\right) x} x^{l_{1}+m_{r}-\frac{1}{2}} \\
& \times K_{k-1-l_{2}}\left(2 \sqrt{\beta_{s} \beta_{r}} x\right) d x
\end{aligned}
$$

In (23), some constant coefficients are omitted. To simplify the calculation, we resort to Fox's $H$ function. Based on [25, eqs.(2.9.4),(2.9.21)], the complementary error function and the exponential function can be rewritten in terms of Fox's $H$ function as

$$
\begin{aligned}
\operatorname{erfc}\left(\sqrt{\frac{b x}{2}}\right) & =\frac{1}{\sqrt{\pi}} H_{1,2}^{2,0}\left[\frac{b x}{2} \mid \begin{array}{c}
(1,1) \\
\left(\frac{1}{2}, 1\right),(0,1)
\end{array}\right] \\
e^{-\left(\frac{b}{2}+\beta_{s}+\beta_{r}\right) x} & =H_{0,1}^{1,0}\left[\left(\frac{b}{2}+\beta_{s}+\beta_{r}\right) x \mid \begin{array}{c}
- \\
(0,1)
\end{array}\right]
\end{aligned}
$$

Then using [26, eq.(2.6.4)], $J_{3}$ can be expressed in terms of the bivariate Fox's function. The average SEP $P_{e 2}$ is given by (26).Finally, combining (22) and (26) yields the average SEP $P_{e}=P_{e 1}-P_{e 2}$. This applies to the scenario where the effect of $Q^{2}(x)$ is can not be ignored, as in quadrature amplitude modulation (QAM). If the quadratic term is not taken into account, $c=0$ will be substituted in (26).

In some special scenarios, AWGN perhaps is not an ideal choice. In [27], AWGGN was proposed for wireless sensor networks and underwater communications due to its versatility in providing a good match to various empirically obtained measurement data. The generalized Gauss distribution is more accurate in describing the noise probability distribution than the traditional Gauss distribution in these scenarios. Therefore, more universal is the analysis of SEP subject to AWGGN, which is given by

$$
P_{e}=\mathrm{E}\left[a Q_{\alpha}(\sqrt{b \gamma})\right]
$$

where $Q_{\alpha}(\cdot)$ is the generalized $Q$ function defined as

$$
Q_{\alpha}(x)=\frac{\Gamma\left(1 / \alpha,\left|\Lambda_{0} x\right|^{\alpha}\right)}{2 \Gamma(1 / \alpha)}
$$

where $\Lambda_{0}=\sqrt{\Gamma(3 / \alpha) / \Gamma(1 / \alpha)}$. It is easily seen that $Q_{\alpha}(x)$ reduces to the traditional $Q$ function when $\alpha=2$. Some common noise types are summarized in [28] with different $\alpha$ values. Applying integration by parts, the SEP subject to AWGGN is given by (29). As a double check, when $\alpha=2$, (29) becomes (22) through some identities, which proves the correctness of the formula.

2) $m$ is a non negative number plus one half: Due to mathematical tractability, we only tackle average SEP when $c=0$ with non negative number $m$. Likewise, using [26, eq.(2.6.2)], the average SEP is given by (30).

Likewise, to analyze the SEP subject to AWGGN, the exact closed form formula is given by (31). Similarly, the SEP expression helps us to accurately assess the joint impact of the noise distribution and channel fading.

Before closing this section, we point out that higher order moments of SNR, ergodic capacity and average SEP are all expressed in exact closed form. All these important performance metrics help us understand the AF relaying system.

\section{Simulation Results}

The correctness of all formulas is checked by computer simulation in this section. According to the location of $\mathrm{S}$, $\mathrm{R}$ and $\mathrm{D}$ with respect to each other and channel qualities, two typical scenarios are considered: all channel gains are 


$$
\begin{aligned}
P_{e 1}= & \mathrm{E}[a Q(\sqrt{b \gamma})]=\frac{a}{2}-\frac{a \sqrt{b} \Gamma\left(m_{d}+\frac{1}{2}\right)}{\sqrt{2 \pi \beta_{d}} \Gamma\left(m_{d}\right)}{ }_{2} F_{1}\left[\frac{1}{2}, m_{d}+\frac{1}{2} ; \frac{3}{2} ; \frac{-b}{2 \beta_{d}}\right]-\frac{a \sqrt{b}}{\sqrt{2} \Gamma\left(m_{r}\right)} \sum_{l_{1}=0}^{m_{s}-1} \sum_{k=0}^{l_{1}} \sum_{l_{2}=0}^{m_{r}-1} \frac{C_{l_{1}}^{k} C_{m_{r}-1}^{l_{2}}}{l_{1} !} \\
& \frac{\beta_{s}^{l_{1}} \beta_{r}^{k-1-l_{2}+m_{r}} 4^{k-l_{2}-1} \Gamma\left(l_{1}+m_{r}-k+l_{2}+\frac{3}{2}\right) \Gamma\left(l_{1}+m_{r}+k-l_{2}-\frac{1}{2}\right)}{\left(\beta_{s}+\beta_{r}+2 \sqrt{\beta_{s} \beta_{r}}+\frac{b}{2}\right)^{l_{1}+m_{r}+k-l_{2}-\frac{1}{2}} \Gamma\left(l_{1}+m_{r}+1\right)} \\
& \times{ }_{2} F_{1}\left[l_{1}+m_{r}+k-l_{2}-\frac{1}{2}, k-l_{2}-\frac{1}{2} ; l_{1}+m_{r}+1 ; \frac{\left(\sqrt{\beta_{s}}-\sqrt{\beta_{r}}\right)^{2}+\frac{b}{2}}{\left(\sqrt{\beta_{s}}+\sqrt{\beta_{r}}\right)^{2}+\frac{b}{2}}\right] \\
& +\frac{a \sqrt{b}}{\sqrt{2} \Gamma\left(m_{r}\right)} \sum_{l_{1}=0}^{m_{s}-1} \sum_{k=0}^{l_{1}} \sum_{l_{2}=0}^{m_{r}-1} \sum_{l_{3}=0}^{m_{d}-1} \frac{C_{l_{1}}^{k} C_{m_{r}-1}^{l_{2}} \beta_{s}^{l_{1}} \beta_{r}^{m_{r}+k-1-l_{2}} \beta_{d}^{l_{3}} 4^{k-1}-l_{2} \Gamma\left(l_{1}+m_{r}+l_{3}+k-l_{2}-\frac{1}{2}\right)}{l_{1} ! l_{3} !\left(\beta_{s}+\beta_{r}+\beta_{d}+\frac{b}{2}+2 \sqrt{\beta_{s} \beta_{r}}\right)^{l_{1}+m_{r}+l_{3}+k-l_{2}-\frac{1}{2}}} \\
& \times \frac{\Gamma\left(l_{1}+m_{r}+l_{3}-k+l_{2}+\frac{3}{2}\right)}{\Gamma\left(l_{1}+m_{r}+l_{3}+1\right)} \\
& \times{ }_{2} F_{1}\left[l_{1}+m_{r}+l_{3}+k-l_{2}-\frac{1}{2}, k-l_{2}-\frac{1}{2} ; l_{1}+m_{r}+l_{3}+1 ; \frac{\beta_{s}+\beta_{r}+\beta_{d}+\frac{b}{2}-2 \sqrt{\beta_{s} \beta_{r}}}{\beta_{s}+\beta_{r}+\beta_{d}+\frac{b}{2}+2 \sqrt{\beta_{s} \beta_{r}}}\right]
\end{aligned}
$$

$$
\begin{aligned}
& P_{e 2}=\mathrm{E}\left[c Q^{2}(\sqrt{b \gamma})\right]=\frac{c}{4}-\frac{c}{2 \sqrt{\pi}} \sum_{l_{3}=0}^{m_{d}-1} \frac{\beta_{d}^{l_{3}} \Gamma\left(2 l_{3}+1\right)_{2} F_{1}\left(l_{3}+\frac{1}{2}, l_{3}+1 ; l_{3}+\frac{3}{2} ;-1-\frac{2 \beta_{d}}{b}\right)}{l_{3} !(2 b)^{l_{3}} \Gamma\left(l_{3}+\frac{3}{2}\right)} \\
& -\frac{\sqrt{b} c}{\sqrt{2} \pi \Gamma\left(m_{r}\right)} \sum_{l_{1}=0}^{m_{s}-1} \sum_{k=0}^{l_{1}} \sum_{l_{2}=0}^{m_{r}-1} \frac{C_{l_{1}}^{k} C_{m_{r}-1}^{l_{2}}}{l_{1} !} \beta_{s}^{\frac{l_{1}-m_{r}-k+l_{2}}{2}+\frac{1}{4}} \beta_{r}^{\frac{m_{r}+k-l_{2}-l_{1}}{2}-\frac{3}{4}} \\
& H_{2,(1: 0), 0,(2: 1)}^{2,0,0,2,1}\left[\begin{array}{c|c}
\frac{b^{2}}{4 \beta_{s} \beta_{r}} & \left(\frac{l_{1}+m_{r}+k-l_{2}}{2}-\frac{1}{4}, 1\right),\left(\frac{l_{1}+m_{r}-k+l_{2}}{2}+\frac{3}{4}, 1\right) \\
\frac{\left(\beta_{s}+\beta_{r}+\frac{b}{2}\right)^{2}}{\beta_{s} \beta_{r}} & (1,2) ;- \\
- & \left(\frac{1}{2}, 2\right),(0,2) ;(0,2)
\end{array}\right] \\
& +\frac{\sqrt{b} c}{\sqrt{2} \pi \Gamma\left(m_{r}\right)} \sum_{l_{1}=0}^{m_{s}-1} \sum_{k=0}^{l_{1}} \sum_{l_{2}=0}^{m_{r}-1} \sum_{l_{3}=0}^{m_{d}-1} \frac{C_{l_{1}}^{k} C_{m_{r}-1}^{l_{2}}}{l_{1} l_{3} !} \beta_{d}^{l_{3}} \beta_{s}^{\frac{l_{1}-m_{r}-l_{3}-k+l_{2}}{2}+\frac{1}{4}} \beta_{r}^{\frac{m_{r}-l_{1}-l_{3}+k-l_{2}}{2}-\frac{3}{4}} \\
& H_{2,(1: 0), 0,(2: 1)}^{2,0,0,2,1}\left[\begin{array}{c|c}
\frac{b^{2}}{4 \beta_{s} \beta_{r}} & \left(\frac{l_{1}+m_{r}+l_{3}+k-l_{2}}{2}-\frac{1}{4}, 1\right),\left(\frac{l_{1}+m_{r}+l_{3}-k+l_{2}}{2}+\frac{3}{4}, 1\right) \\
\frac{\left(\beta_{s}+\beta_{r}+\beta_{d}+\frac{b}{2}\right)^{2}}{\beta_{s} \beta_{r}} & (1,2) ;- \\
- & \left(\frac{1}{2}, 2\right),(0,2) ;(0,2)
\end{array}\right]
\end{aligned}
$$

$$
\begin{aligned}
& P_{e}=\mathrm{E}\left[a Q_{\alpha}(\sqrt{b \gamma})\right]=\int_{0}^{\infty} \frac{a \alpha \Lambda_{0} \sqrt{b}}{4 \Gamma(1 / \alpha) \sqrt{z}} e^{-\left(\sqrt{b z} \Lambda_{0}\right)^{\alpha}} F_{\gamma}(z) d z=\frac{a}{2}-\frac{a \sqrt{b} \Lambda_{0}}{4 \Gamma(1 / \alpha) \sqrt{\beta_{d}} \Gamma\left(m_{d}\right)} \\
& \times H_{2,2}^{1,2}\left[\frac{\sqrt{b} \Lambda_{0}}{\sqrt{\beta_{d}}} \mid \begin{array}{c}
\left(\frac{1}{2}-m_{d}, \frac{1}{2}\right),\left(\frac{1}{2}, \frac{1}{2}\right) \\
\left(0, \frac{1}{\alpha}\right),\left(-\frac{1}{2}, \frac{1}{2}\right)
\end{array}\right]-\frac{a \sqrt{b} \Lambda_{0}}{\Gamma(1 / \alpha) \Gamma\left(m_{r}\right)} \sum_{l_{1}=0}^{m_{s}-1} \sum_{k=0}^{l_{1}} \sum_{l_{2}=0}^{m_{r}-1} \frac{C_{l_{1}}^{k} C_{m_{r}-1}^{l_{2}} \beta_{s}^{\frac{l_{1}-m_{r}-k+l_{2}}{2}+\frac{1}{4}} \beta_{r}^{\frac{k-l_{2}-l_{1}+m_{r}}{2}-\frac{3}{4}}}{l_{1} !} \\
& \times H_{2,[0: 0], 0,[1: 1]}^{2,0,0,1,1}\left[\begin{array}{c|c}
\frac{\left(\beta_{s}+\beta_{r}\right)^{2}}{\beta_{s} \beta_{r}} & \left(\frac{l_{1}+m_{r}+k-l_{2}}{2}-\frac{1}{4}, 1\right),\left(\frac{l_{1}+m_{r}-k+l_{2}}{2}+\frac{3}{4}, 1\right) \\
\frac{b^{2} \Lambda_{0}^{4}}{\beta_{s} \beta_{r}} & - \\
- & (0,2) ;\left(0, \frac{4}{\alpha}\right)
\end{array}\right] \\
& +\frac{a \sqrt{b} \Lambda_{0}}{\Gamma(1 / \alpha) \Gamma\left(m_{r}\right)} \sum_{l_{1}=0}^{m_{s}-1} \sum_{k=0}^{l_{1}} \sum_{l_{2}=0}^{m_{r}-1} \sum_{l_{3}=0}^{m_{d}-1} \frac{C_{l_{1}}^{k} C_{m_{r}-1}^{l_{2}} \beta_{s}^{\frac{l_{1}-m_{r}-k+l_{2}-l_{3}}{2}+\frac{1}{4}} \beta_{r}^{\frac{k-l_{2}-l_{1}+m_{r}-l_{3}}{2}-\frac{3}{4}} \beta_{d}^{l_{3}}}{l_{1} ! l_{3} !} \\
& \times H_{2,(0: 0), 0,(1: 1)}^{2,0,0,1,1}\left[\begin{array}{c|c}
\frac{\left(\beta_{s}+\beta_{r}+\beta_{d}\right)^{2}}{\beta_{s} \beta_{r}} & \left(\frac{l_{1}+m_{r}+l_{3}+k-l_{2}}{2}-\frac{1}{4}, 1\right),\left(\frac{l_{1}+m_{r}+l_{3}-k+l_{2}}{2}+\frac{3}{4}, 1\right) \\
\frac{b^{2} \Lambda_{0}^{4}}{\beta_{s} \beta_{r}} & -;- \\
- & (0,2) ;\left(0, \frac{4}{\alpha}\right)
\end{array}\right]
\end{aligned}
$$




$$
\begin{aligned}
& P_{e}=\mathrm{E}[a Q(\sqrt{b \gamma})]=\frac{a}{2}-\frac{a \sqrt{b} \Gamma\left(m_{d}+\frac{1}{2}\right)}{\sqrt{2 \pi \beta_{d}} \Gamma\left(m_{d}\right)}{ }_{2} F_{1}\left(\frac{1}{2}, m_{d}+\frac{1}{2} ; \frac{3}{2} ; \frac{-b}{2 \beta_{d}}\right)-\frac{a \sqrt{b}\left(\sqrt{\beta_{s}}+\sqrt{\beta_{r}}\right)}{\sqrt{2 \pi} \Gamma\left(m_{s}\right) \Gamma\left(m_{r}\right)} \sum_{k_{1}=0}^{\left\lfloor m_{s}\right\rfloor} \sum_{k_{2}=0}^{\left\lfloor m_{r}\right\rfloor} \frac{\left(\left\lfloor m_{s}\right\rfloor+k_{1}\right) !}{4^{k_{1}+k_{2}} k_{1} ! k_{2} !} \\
& \times \frac{\left(\left\lfloor m_{r}\right\rfloor+k_{2}\right) ! \beta_{s}^{\frac{m_{s}}{2}-\frac{1}{4}-\frac{k_{1}}{2}} \beta_{r}^{\frac{m_{r}}{2}-\frac{1}{4}-\frac{k_{2}}{2}}}{\left(\left\lfloor m_{s}\right\rfloor-k_{1}\right) !\left(\left\lfloor m_{r}\right\rfloor-k_{2}\right) !} \Gamma\left(\frac{m_{s}-k_{1}+m_{r}-k_{2}+1}{2}\right)\left[\frac{b}{2}+\left(\sqrt{\beta_{s}}+\sqrt{\beta_{r}}\right)^{2}\right]^{\frac{k_{1}-m_{s}+k_{2}-m_{r}-1}{2}} \\
& +\frac{a}{2 \Gamma\left(m_{s}\right) \Gamma\left(m_{r}\right) \Gamma\left(m_{d}\right)} \sum_{k_{1}=0}^{\left\lfloor m_{s}\right\rfloor} \sum_{k_{2}=0}^{\left\lfloor m_{r}\right\rfloor} \sum_{l=0}^{\left.\frac{\left\lfloor m_{s}-k_{1}+m_{r}-k_{2}\right.}{2}\right\rfloor} \frac{\left(\left\lfloor m_{s}\right\rfloor+k_{1}\right) !\left(\left\lfloor m_{r}\right\rfloor+k_{2}\right) ! \beta_{s}^{\frac{m_{s}}{2}-\frac{1}{4}-\frac{k_{1}}{2}}}{4^{k_{1}+k_{2}} k_{1} ! k_{2} !\left(\left\lfloor m_{s}\right\rfloor-k_{1}\right) !\left(\left\lfloor m_{r}\right\rfloor-k_{2}\right) ! 2^{2 l}} \\
& \times \frac{\beta_{r}^{\frac{m_{r}}{2}-\frac{1}{4}-\frac{k_{2}}{2}}\left(m_{s}-k_{1}+m_{r}-k_{2}\right) !(-1)^{l}}{l !\left(m_{s}-k_{1}+m_{r}-k_{2}-2 l\right) !}\left(\sqrt{\beta_{s}}+\sqrt{\beta_{r}}\right)^{k_{1}-m_{s}+k_{2}-m_{r}+1} \\
& \times H_{1,(1: 1), 0,(2: 2)}^{1,0,0,2,2}\left[\begin{array}{c|c}
\left.\frac{1}{2}, 1\right) \\
\frac{2 \beta_{d}}{b} & (1,1) ;(1,1) \\
\frac{2\left(\beta_{s}+\beta_{r}\right)^{2}}{b} & - \\
& \left(m_{d}, 1\right),(0,1) ;\left(m_{s}-k_{1}+m_{r}-k_{2}-l-\frac{1}{2}, 1\right),(0,1)
\end{array}\right]
\end{aligned}
$$

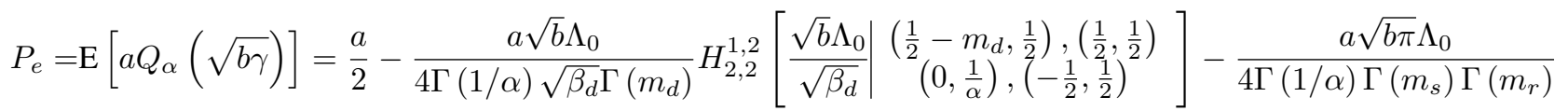

$$
\begin{aligned}
& \sum_{k_{1}=0}^{\left\lfloor m_{s}\right\rfloor} \sum_{k_{2}=0}^{\left\lfloor m_{r}\right\rfloor} \sum_{l=0}^{\left\lfloor\frac{m_{s}-k_{1}+m_{r}-k_{2}}{2}\right\rfloor} \frac{\left(\left\lfloor m_{s}\right\rfloor+k_{1}\right) !\left(\left\lfloor m_{r}\right\rfloor+k_{2}\right) ! \beta_{s}^{\frac{m_{s}}{2}-\frac{1}{4}-\frac{k_{1}}{2}}}{4^{k_{1}+k_{2}} k_{1} ! k_{2} !\left(\left\lfloor m_{s}\right\rfloor-k_{1}\right) !\left(\left\lfloor m_{r}\right\rfloor-k_{2}\right) ! 2^{2 l}} \\
& \times \frac{\beta_{r}^{\frac{m_{r}}{2}-\frac{1}{4}-\frac{k_{2}}{2}}\left(m_{s}-k_{1}+m_{r}-k_{2}\right) !(-1)^{l}}{l !\left(m_{s}-k_{1}+m_{r}-k_{2}-2 l\right) !}\left(\sqrt{\beta_{s}}+\sqrt{\beta_{r}}\right)^{k_{1}-m_{s}+k_{2}-m_{r}} \\
& \times H_{2,2}^{1,2}\left[\begin{array}{c|c}
\sqrt{b} \Lambda_{0} & \left(1-m_{s}+k_{1}-m_{r}+k_{2}+l, \frac{1}{2}\right),\left(\frac{1}{2}, \frac{1}{2}\right) \\
\sqrt{\beta_{s}}+\sqrt{\beta_{r}} & \left(0, \frac{1}{\alpha}\right),\left(-\frac{1}{2}, \frac{1}{2}\right)
\end{array}\right] \\
& +\frac{a \sqrt{\pi}}{2 \Gamma(1 / \alpha) \Gamma\left(m_{s}\right) \Gamma\left(m_{r}\right) \Gamma\left(m_{d}\right)} \sum_{k_{1}=0}^{\left\lfloor m_{s}\right\rfloor} \sum_{k_{2}=0}^{\left\lfloor m_{r}\right\rfloor} \sum_{l=0}^{\left\lfloor\frac{m_{s}-k_{1}+m_{r}-k_{2}}{2}\right\rfloor} \frac{\left(\left\lfloor m_{s}\right\rfloor+k_{1}\right) !\left(\left\lfloor m_{r}\right\rfloor+k_{2}\right) ! \beta_{s}^{\frac{m_{s}}{2}-\frac{1}{4}-\frac{k_{1}}{2}}}{4^{k_{1}+k_{2}} k_{1} ! k_{2} !\left(\left\lfloor m_{s}\right\rfloor-k_{1}\right) !\left(\left\lfloor m_{r}\right\rfloor-k_{2}\right) ! 2^{2 l}} \\
& \times \frac{\beta_{r}^{\frac{m_{r}}{2}-\frac{1}{4}-\frac{k_{2}}{2}}\left(m_{s}-k_{1}+m_{r}-k_{2}\right) !(-1)^{l}}{l !\left(m_{s}-k_{1}+m_{r}-k_{2}-2 l\right) !}\left(\sqrt{\beta_{s}}+\sqrt{\beta_{r}}\right)^{k_{1}-m_{s}+k_{2}-m_{r}+1} \\
& \times H_{1,(1: 1), 0,(2: 2)}^{1,0,0,2,2}\left[\begin{array}{c|c}
\frac{\beta_{d}}{b \Lambda_{0}^{2}} & \left(\frac{1}{\alpha}, \frac{2}{\alpha}\right) \\
\frac{\left(\beta_{s}+\beta_{r}\right)^{2}}{b \Lambda_{0}^{2}} & (1,1) ;(1,1) \\
& - \\
& \left(m_{d}, 1\right),(0,1) ;\left(m_{s}-k_{1}+m_{r}-k_{2}-l-\frac{1}{2}, 1\right),(0,1)
\end{array}\right]
\end{aligned}
$$

normalized to unity; the channel gains of the $\mathrm{S} \rightarrow \mathrm{R}$ and $\mathrm{R} \rightarrow \mathrm{D}$ links are normalized to unity while the channel gain of the $\mathrm{S} \rightarrow \mathrm{D}$ link is only one tenth, referred to as weak SD. This corresponds to a scenario where the destination node is far from the source node or the user is at the cell edge. The noise variances of all receivers are equal $\sigma_{r}^{2}=\sigma_{d}^{2}=\sigma^{2}$. The source node and the relay use the same transmission power $P_{s}=P_{r}$. The average SNR per hop is defined as $P_{s} / \sigma^{2}$.

Figs. 2 and 3 show that the first order moment of SNR is consistently growing. The simulated and computed curves agree very well, validating the accuracy of our analysis. As can be seen from both figures, there is always a gap between a strong SD channel and a weak SD channel. Other higher order moments can be similarly drawn using eqs.(10) and (11). Using the first and second moment, the amount of fading can easily be evaluated by $\mathrm{E}\left(\gamma^{2}\right) / \mathrm{E}^{2}(\gamma)-1$.

The variation of the ergodic capacity with average SNR per hop is shown in Figs.4 and 5, where theoretical results are consistent with simulated curves. The larger fading parameter offers performance improvement over the smaller one. When $m_{s}=m_{r}=m_{d}=0.5$, in high SNR region, the capacity is 4.1912 at $28 \mathrm{~dB}$ and 4.5204 at $30 \mathrm{~dB}$. This implies that the multiplexing gain for a non negative integer $m$ is

$$
\begin{gathered}
\frac{10 \times \lg 2 \times(4.5204-4.1912)}{30-28} \\
=0.4955 \approx \frac{1}{2}
\end{gathered}
$$

While for $m_{s}=m_{r}=0.5, m_{d}=1.5$, the capacity is 4.4736 


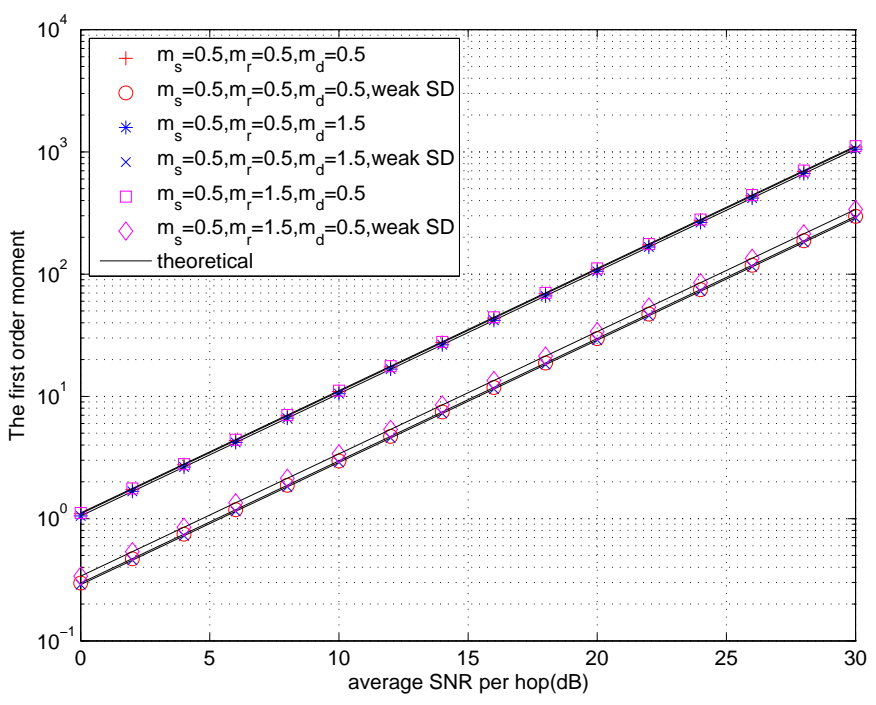

Figure 2. Comparison of ergodic capacity with non negative integer plus one half parameters

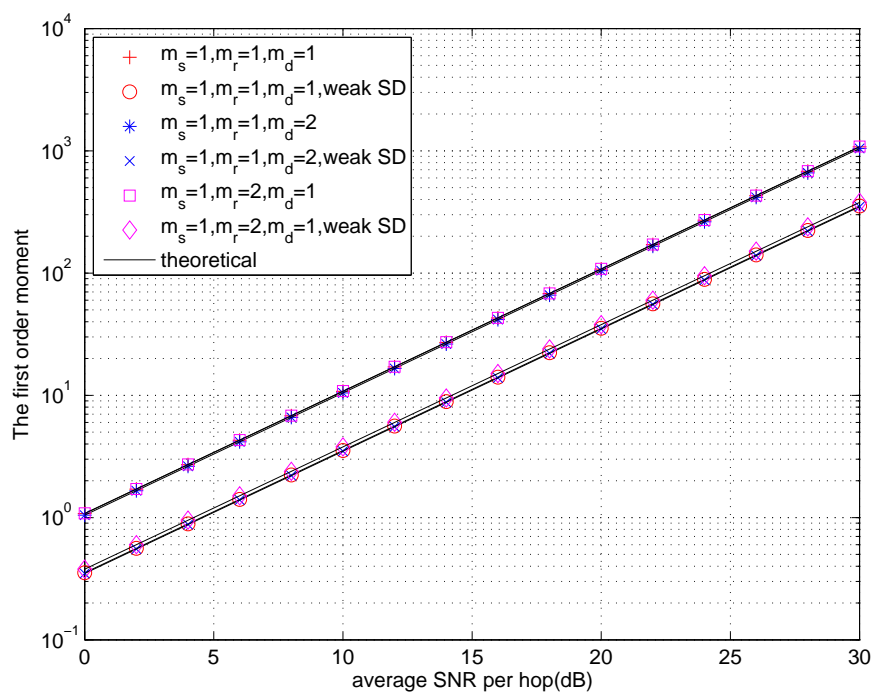

Figure 3. Comparison of average SNR with positive integer parameters

at $28 \mathrm{~dB}$ and 4.8049 at $30 \mathrm{~dB}$. The multiplexing gain becomes

$$
\begin{gathered}
\frac{10 \times \lg 2 \times(4.8049-4.4736)}{30-28} \\
=0.4987 \approx \frac{1}{2}
\end{gathered}
$$

A similar phenomenon is observed in Fig.5 when $m$ is a positive integer. The ergodic capacity increases asymptotically in a linear manner with a slope $1 / 2$. The case of AF relaying offers a multiplexing gain of $1 / 2$, half the multiplexing gain in conventional peer to peer communications.

Figs. (6) and (7) shows the average SEP for 4-ary pulse amplitude modulation (4PAM) and 4QAM, respectively. Here, two different modulation schemes are due to different expressions eqs.(22), (26) and (30). From Fig.6, when $m_{s}=m_{r}=$ $m_{d}=0.5$, in the high SNR region, the SEP is 0.0037466 at average SNR per hop $=28 \mathrm{~dB}$ and 0.0023726 at average SNR per hop $=30 \mathrm{~dB}$. This implies that the diversity gain for non

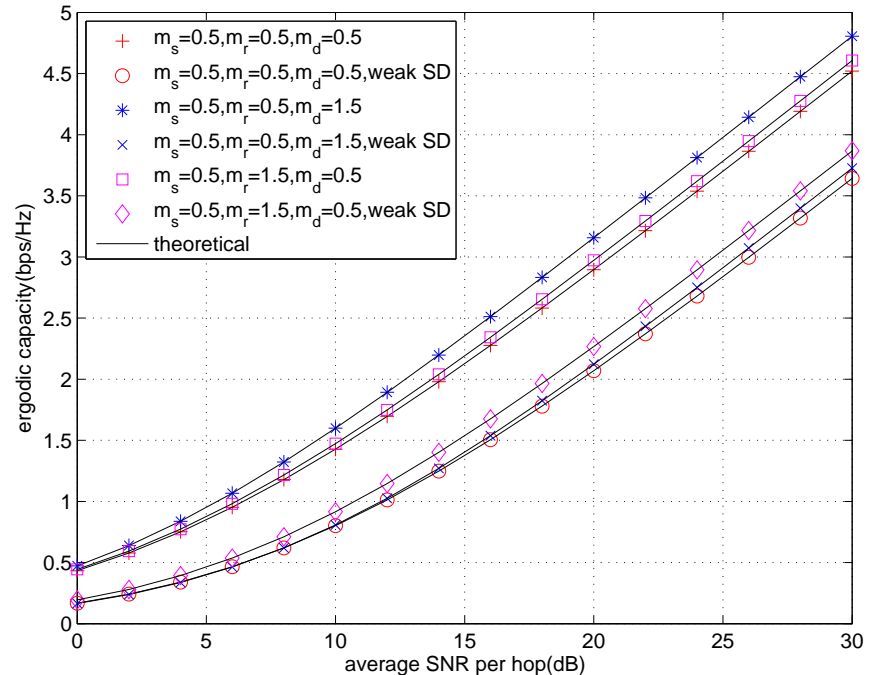

Figure 4. Comparison of ergodic capacity with non negative integer plus one half parameters

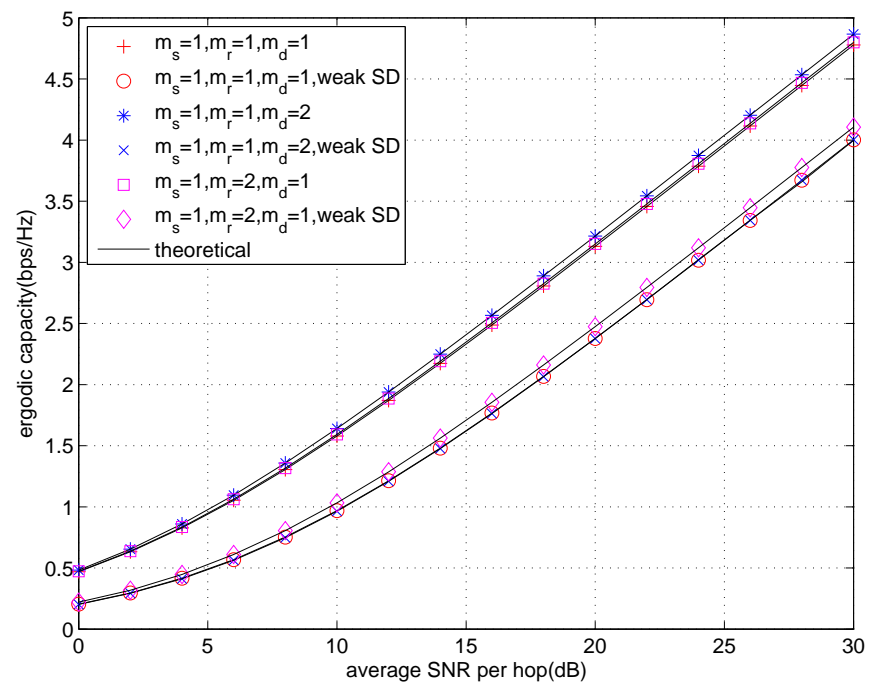

Figure 5. Comparison of ergodic capacity with positive integer parameters

negative integer $m$ is [29]

$$
\begin{aligned}
& \frac{10 \lg (0.0037466 / 0.0023726)}{30-28} \\
= & 0.9921 \approx \min \left(m_{s}, m_{r}\right)+m_{d}
\end{aligned}
$$

While for $m_{s}=m_{r}=0.5, m_{d}=1.5$, the SEP is $7.5603 \times$ $10^{-5}$ at $28 \mathrm{~dB}$ and $3.0259 \times 10^{-5}$ at $30 \mathrm{~dB}$. The diversity gain becomes

$$
\begin{gathered}
\frac{10 \lg (7.5603 / 3.0259)}{30-28} \\
=1.9884 \approx \min \left(m_{s}, m_{r}\right)+m_{d}
\end{gathered}
$$

A similar phenomenon is observed in Fig.7 when $m$ is a positive integer. Therefore, a key conclusion is reached: the diversity gain for a selection combining relaying system in Nakagami fading channel is $\min \left(m_{s}, m_{r}\right)+m_{d}$. The reason is that the error probability of the $\mathrm{S} \rightarrow \mathrm{R}$ and $\mathrm{R} \rightarrow \mathrm{D}$ links is limited to the smaller channel quality due to the inherent 


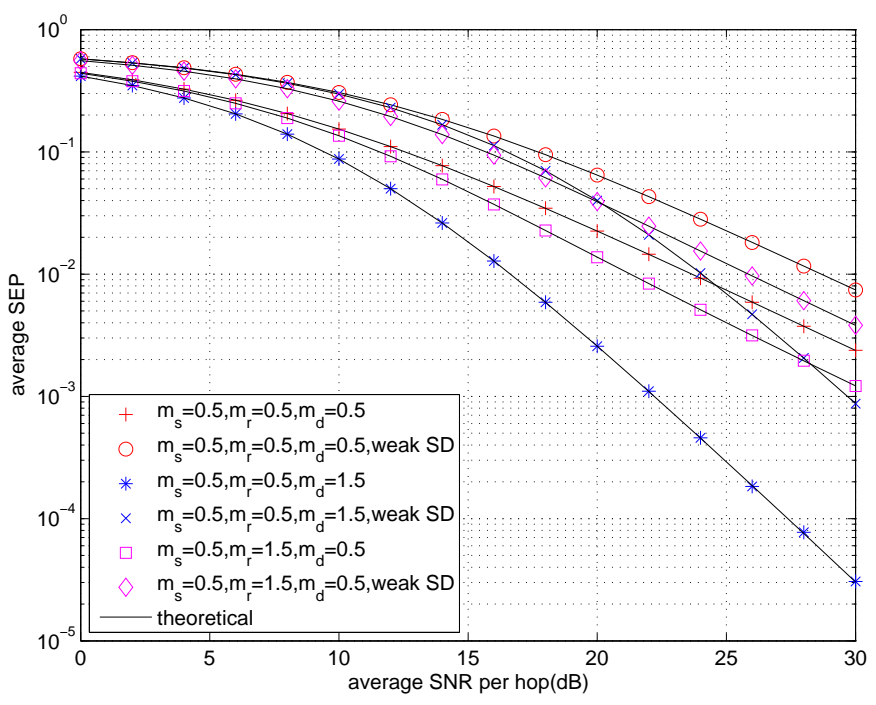

Figure 6. SEP of pulse amplitude modulation (4PAM) with non negative integer plus one half parameters

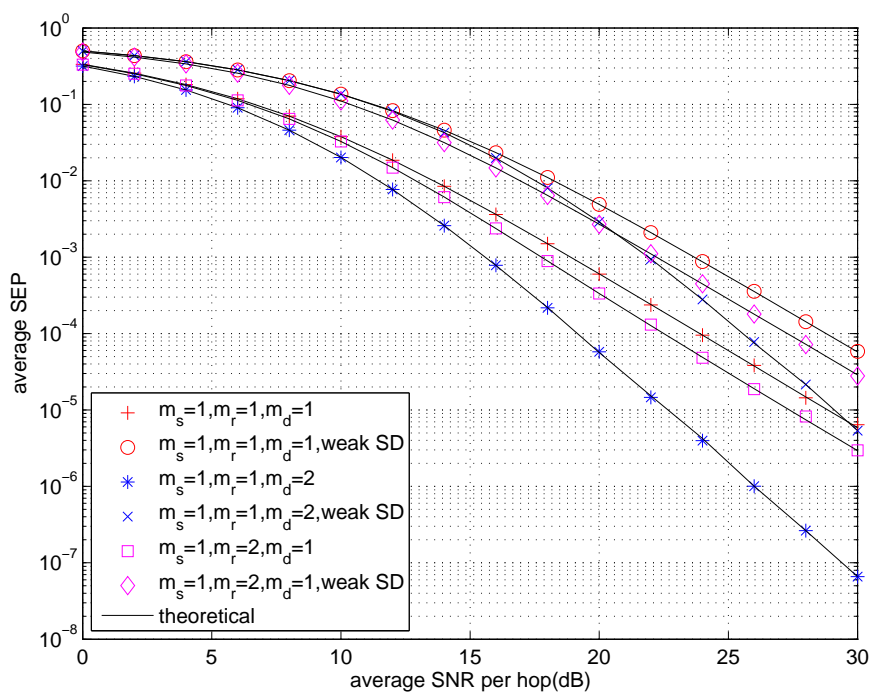

Figure 7. SEP of 4QAM with positive integer parameters

property of relay technology, while the whole error probability of $\mathrm{S} \rightarrow \mathrm{R} \rightarrow \mathrm{D}$ and $\mathrm{S} \rightarrow \mathrm{D}$ links is limited to the larger channel quality because of the decision characteristic of selection combining.

\section{CONClusion}

Due to low complexity of selection combining, we analyzed its performance in an AF relaying system over a Nakagami- $m$ channel. All derived exact formulas are expressed in closed form using special functions. In addition, the AWGN case is extended to AWGGN, whose practical importance is often overlooked. We analyzed the multiplexing gain and diversity gain which can approximately predict the asymptotic behavior in the high SNR regime. Simulation results for various fading parameters and channel qualities confirm the accuracy of the analysis.

\section{REFERENCES}

[1] Fuhui Zhou, Norman C. Beaulieu, Julian Cheng, Zheng Chu and Yuhao Wang, "Robust max-min fairness resource allocation in sensing-based wideband cognitive radio with SWIPT: imperfect channel sensing," IEEE Systems Journal, accepted, 2017.

[2] Xinyu Zhang, Nan Zhao, F. Richard Yu, Minglu Jin and Victor C. M. Leung, "Resource allocation in topology management of asymmetric interference networks," IEEE Systems Journal, vol. 12, no. 1, pp. 9931003, Mar. 2018

[3] Devendra S. Gurjar, Ha H. Nguyen and Hoang D. Tuan, "Wireless information and power transfer for IoT applications in overlay cognitive radio networks," IEEE Internet Things J., accepted, 2018.

[4] M. Bilim, N. Kapucu and I. Develi, "A closed-form approximate BEP expression for cooperative IDMA systems over multipath Nakagami- $m$ fading channels," IEEE Commun. Lett., vol. 20, no. 8, pp. 1599-1602, Aug. 2016.

[5] J. M. Moualeu, W. Hamouda and F. Takawira, "Relay selection for coded cooperative networks with outdated CSI over Nakagami- $m$ fading channels," IEEE Trans. Wireless Commun., vol. 13, no. 5, pp. 2362-2373, May 2014.

[6] J. Shi, C. Dong and L. L. Yang,"Performance comparison of cooperative relay links with different relay processing strategies: Nakagami / Gamma approximation approaches," Eurasip J. Wireless Commun. Netw., vol. 2014, pp. 1-17, 2014.

[7] S. I. Chu, "Outage probability and dmt performance of underlay cognitive networks with incremental DF and AF relaying over Nakagami- $m$ fading channels," IEEE Commun. Lett., vol. 18, no. 1, pp. 62-65, Jan. 2014.

[8] T. Sanguanpuak, N. Rajatheva and A. Taparugssanagorn, "Performance analysis of OSTBC for partial relay selection with correlated antennas over Nakagami-m fading," IEEE Wireless Commun. Lett., vol. 2, no. 3, pp. 355-358, Jun. 2013.

[9] P. Som and A. Chockalingam, "Bit error probability analysis of SSK in DF relaying with threshold-based best relay selection and selection combining," IEEE Commun. Lett., vol. 18, no. 1, pp. 18-21, Jan. 2014.

[10] A. B. Sediq and H. Y.anikomeroglu, "Performance analysis of selection combining of signals with different modulation levels in cooperative communications," IEEE Trans. Veh. Technol., vol. 60, no. 4, pp. 18801887, May 2011.

[11] H. X. Nguyen, "Selection combining for noncoherent decode-andforward relay networks," Eurasip J. Wireless Commun. Netw., vol. 2011, pp. 1-10, 2011.

[12] V. N. Q. Bao and H. Y. Kong, "An exact closed-form expression for bit error rate of decode-and-forward relaying using selection combining over Rayleigh fading channels," J. Commun. Netw., vol. 11, no. 5, pp. 480-488, Oct. 2009

[13] K. J. Kim, T. Q. Duong and H. V. Poor, "Outage probability of singlecarrier cooperative spectrum sharing systems with decode-and-forward relaying and selection combining," IEEE Trans. Wireless Commun., vol. 12, no. 2, pp. 806-817, Feb. 2013.

[14] Yansha Deng, Lifeng Wang, Maged Elkashlan, Kyeong Jin Kim, and Trung Q. Duong, "Generalized selection combining for cognitive relay networks over Nakagami-m fading," IEEE Trans. Signal Process., vol. 63, no. 8, pp. 1993-2006, Apr. 2015.

[15] Feng Xu, Francis C. M. Lau, Qing F. Zhou, and Dian-Wu Yue, "Outage performance of cooperative communication systems using opportunistic relaying and selection combining receiver," IEEE Signal Process. Lett., vol. 16 , no. 4 , pp. 237-240, Apr. 2009.

[16] Ehsan Soleimani-Nasab, Mehrdad Ardebilipour and Ashkan Kalantari, "Performance analysis of selective combining decode-and-forward relay networks over Nakagami- $n$ and Nakagami- $q$ fading channels," Wireless Commun. Mob. Comput., vol. 14, no. 16, pp. 1564-1581, Nov. 2014.

[17] M. R. Avendi and Ha H. Nguyen, "Performance of selection combining for differential amplify-and-forward relaying over time-varying channels," IEEE Trans. Wireless Commun., vol. 13, no. 8, pp. 4156-4166, Aug. 2014.

[18] Yi Lou, Qi-Yue Yu, Julian Cheng and Hong-Lin Zhao, "Exact BER analysis of selection combining for differential SWIPT relaying systems," IEEE Signal Process. Lett., vol. 24, no. 8, pp. 1198-1202, Aug. 2017.

[19] M. R. Avendi and Ha H. Nguyen, "Selection combining for differential amplify-and-forward relaying over Rayleigh-fading channels," IEEE Signal Process. Lett., vol. 20, no. 3, pp. 277-280, Mar. 2013.

[20] S.N. Datta, S. Chakrabarti and R. Roy, "Comprehensive error performance analysis of distributed selection combining with multi-antenna amplify-and-forward relay over Nakagami- $m$ fading channels," Electron. Lett., vol. 46, no. 22, pp. 1523-1525, Nov. 2010. 
[21] Shao-I Chu, "Performance of amplify-and-forward cooperative diversity networks with generalized selection combining over Nakagami- $m$ fading channels," IEEE Commun. Lett., vol. 16, no. 5, pp. 634-637, May 2012.

[22] Michelle S. P. Facina, Haniph A. Latchman, H. Vincent Poor and Moises V. Ribeiro, "Cooperative in-home power line communication: analyses based on a measurement campaign," IEEE Trans. Commun., vol. 64, no. 2, pp. 778-789, Feb. 2016.

[23] I. S. Gradshteyn and I. M. Ryzhik, Table of integrals, series and products. Academic Press, 2007, 7th edition.

[24] Jing Yang, Pingzhi Fan, Trung Q. Duong, and Xianfu Lei, "Exact performance of two-way AF relaying in Nakagami- $m$ fading environment," IEEE Trans. Wireless Commun., vol. 10, no. 3, pp. 980-987, Mar. 2011.

[25] A. A. Kilbas and M. Saigo, $H$-transforms: theory and applications. CRC Press, 2004.

[26] A. M. Mathai and R. K. Saxena, The $H$-function with applications in statistics and other disciplines. John Wiley \& Sons, 1978.

[27] H. Soury, F. Yilmaz and M. S. Alouini, "Average bit error probability of binary coherent signaling over generalized fading channels subject to additive generalized Gaussian noise," IEEE Commun. Lett., vol. 16, no. 6, pp. 785-788, Jun. 2012.

[28] H. Soury, F. Yilmaz and M. S. Alouini, "Error rates of M-PAM and M-QAM in generalized fading and generalized Gaussian noise environments," IEEE Commun. Lett., vol. 17, no. 10, pp. 1932-1935, Oct. 2013.

[29] M. D. Selvaraj and Ranjan K. Mallik, "Performance of full CSI selection combining for cooperative diversity systems," IEEE Trans. Commun., vol. 60, no. 9, pp. 2482-2488, Sep. 2012. 